\title{
Can histologic remission be a better prognostic factor and therapeutic target beyond endoscopic mucosal healing in patients with ulcerative colitis?
}

\author{
Hyo Jun Ahn, Sang-Bum Kang \\ Division of Gastroenterology, Department of Internal Medicine, Daejeon St. Mary's Hospital, College of Medicine, The Catholic University of \\ Korea, Daejeon, Korea
}

\section{Article: Association of endoscopic and histological remission with clinical course in patients of ulcerative colitis (Intest Res 2018;16:55-61)}

Since the development of the "treat to target" and "mucosal healing" concepts, the treatment goal for patients with UC has changed. Before the mucosal healing concept emerged, the therapeutic target of UC was simply to control symptoms such as rectal bleeding and stool frequency. In recent years, the achievement of mucosal healing indicates a better prognosis in patients with UC. Practically, mucosal healing has been associated with prolonged clinical remission, lower hospitalization rates, a lower risk of the development of colorectal cancer, and a lower risk of colectomy. Consensus guidelines for clinical practice and trial endpoints in UC recommend that clinicians achieve endoscopic mucosal healing beyond the resolution of clinical symptoms. In addition, "deep remission," defined as the combination of clinical remission and endoscopic mucosal healing, has been suggested as a treatment target in the era of biological therapy for IBD.

Furthermore, recent studies have shown that histological healing beyond endoscopic mucosal healing can lead to a better prognosis in patients with UC. According to these studies, histologic inflammation in patients with UC increases the risk of colorectal neoplasia, ${ }^{1,2}$ whereas histologic

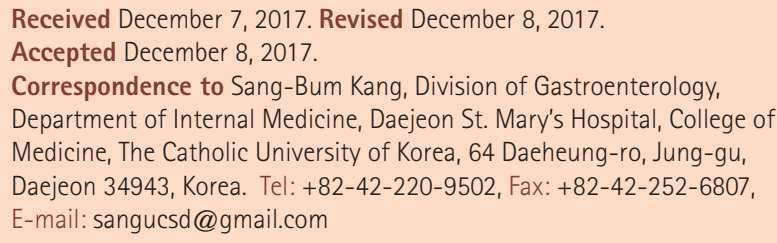

healing can prevent clinical recurrence, reduce steroid use, and lower hospitalization rates. ${ }^{3,4}$ Meanwhile, persistent histologically active disease is suggested to be common despite endoscopic mucosal healing. Although UC patients who achieve endoscopic mucosal healing have a favorable prognosis, nearly $40 \%$ of them have chronic persistent inflammatory activity on histological examination. There have been few systematic analyses of the concordance between histological indices for remission in UC.

The current issue of Intestinal Research includes a report by Narang et al. ${ }^{5}$ describing a prospective observational study emphasizing the importance of histologic remission in UC over endoscopic remission. In this study, among the total 46 patients who achieved endoscopic remission for at least 6 months, 4 endoscopic biopsies from the rectosigmoid regions were performed. Per Geboes criteria, ${ }^{6}$ grade $0 / 1$ was labeled histological remission. As a result, histologic remission was noted in $67.3 \%(31 / 46)$ of the patients, whereas $32.7 \%(15 / 46)$ still had histologically active disease in the form of eosinophil and neutrophil infiltration into the lamina propria $(13 / 15,86.6 \%)$, cryptitis $(14 / 15,93.3 \%)$, or a crypt abscess $(8 / 15,53.3 \%)$. In addition, after a 1-year follow-up, the sustained clinical remission rate was significantly higher in patients with histologic remission than in those with histologically active disease ( $87.1 \%$ vs. $46.6 \%, P=0.006)$. In multivariate analyses, relapses depended upon the histological score $(P<0.001)$ but not on patient factors like age, sex, duration of clinical remission, disease extent, or Mayo endo-

\footnotetext{
๑ Copyright 2018. Korean Association for the Study of Intestinal Diseases. All rights reserved.

This is an Open Access article distributed under the terms of the Creative Commons Attribution Non-Commercial License (http://creativecommons.org/licenses/by-nc/4.0)

which permits unrestricted non-commercial use, distribution, and reproduction in any medium, provided the original work is properly cited.
} 
scopic score. However, the presence of basal lymphoid aggregates or basal plasmacytosis had no clinical significance $(P=1.000)$. This finding is different from those of previous reports, but a recent study by Mosli et al. ${ }^{7}$ showed that basal plasmacytosis had the lowest intraclass correlation coefficient. Thus, scoring basal plasmacytosis using the histologic index may be less sensitive.

The current study was the first report from an Asian country of the discrepancy between endoscopic and histologic remission. It revealed that histologic remission serves as a better endpoint than endoscopic remission of sustained remission in UC patients. The limitations of this study are its small sample size and non-validated histologic index. Accordingly, multicenter prospective studies including large numbers of patients are needed to ascertain histologic remission as a crucial marker of the prognosis of UC patients in Asia.

Because the histological features of UC vary by disease stage, histological scoring systems are needed to identify the histological activity and many histological indices have been developed and used. Various histological scoring systems have been created since Truelove and Richards first introduced a histological scoring system for patients with UC in 1956. Although the scoring system created by Geboes et al. ${ }^{6}$ (Geboes index) has been the most commonly used for histological assessments in many clinical trials, it has some validation-related limitations.

According to the Cochrane review by Mosli et al., ${ }^{8}$ there are currently 30 UC histology scoring indices. Some of these include the degree of acute or chronic inflammation, inflammatory cell infiltration within the lamina propria and epithelium, architectural distortion, and the integrity of the colonic epithelium as histological activity parameters. Only 11 of these scoring indices have been validated. Among them, the Nancy histological index (NHI) and the Robarts histopathology index (RHI) have been used for validation and feature reliability, content validity, construct validity, and criterion validity. However, none of the histologic scoring indices for UC have been fully validated. Recently, Marchal-Bressenot et al. ${ }^{9}$ developed a validated index for assessing histological disease activity in UC, namely, the NHI.

The NHI is composed of 3 histological items defining 5 grades of disease activity: absence of significant histological disease (grade 0 ), chronic inflammatory infiltration with no acute inflammatory infiltrates (grade 1), mildly active disease (grade 2), moderately active disease (grade 3 ), and severely active disease (grade 4). In addition, the RHI was developed by Mosli et al. ${ }^{7}$ and is highly validated. The RHI consists of 4 component items: chronic inflammatory infiltrate (4 levels), lamina propria neutrophils (4 levels), neutrophils in the epithelium (4 levels), and erosion or ulceration (4 levels). The total score ranges from 0 (no disease activity) to 33 (severe disease activity). Nevertheless, these 2 new histological indices were not fully validated and have some limitations such as the lack of a prognostic value, generalization, and prediction of therapeutic responses. Moreover, Christensen et al. ${ }^{10}$ recently reported that histologic normalization is associated with improved clinical outcomes compared with histologic quiescence and activity and is more predictive of improved outcomes than endoscopic mucosal healing alone or histologic quiescence alone, although only $10 \%$ of patients had complete histologic normalization in all segments. In this study, patients were categorized into histologic normalization, histologic quiescence, and active disease groups. The histological findings were compared to the clinical and endoscopic findings. The authors of this study propose that a histologic assessment of disease activity should be part of the endoscopic assessment in cases of IBD.

In response to previous studies, the trend of current treatment is shifting to histologic remission beyond endoscopic mucosal healing. Nevertheless, there are various limitations regarding whether histologic remission should be the treatment target in clinical practice. First, the definition of histologic remission has not yet been established. Although many different studies have set histologic remission as the primary goal, each defined it slightly differently. Next, the histological index has not yet been fully validated and is not globally standardized, although 2 novel histologic indices were recently developed and validated. However, these indices have not yet been applied in large-scale clinical studies and their prognostic value has not been determined. Further, the technical aspects of optimizing histologic investigations should be considered.

A globally standardized consensus is also needed for the number of tissues required for the optimized examination, anatomical location of the tissue sampling, and timing of tissue collection after treatment. Finally, the question persists about how the concept of histologic remission should be applied in clinical practice. Endoscopic mucosal healing cannot be achieved in all UC patients; furthermore, it is more difficult to achieve histologic remission. Thus, the question remains about whether to increase or change medications in asymptomatic UC patients with mucosal healing if histologic inflammation persists. Therefore, it is not currently practical to establish histologic remission as a therapeutic goal in all UC patients. In the future, many clinical studies should clari- 
fy which patients should be treated with histologic remission as the therapeutic target. In addition, the role of histologic remission in determining the efficacy of various medications should be further investigated.

\section{FINANCIAL SUPPORT}

The authors received no financial support for the research, authorship, and/or publication of this article.

\section{CONFLICT OF INTEREST}

No potential conflict of interest relevant to this article was reported.

\section{AUTHOR CONTRIBUTION}

H.J.A., original draft; S.B.K., review and editing. All authors read and approved the final version of the manuscript.

\section{REFERENCES}

1. Bopanna S, Roy M, Das P, et al. Role of random biopsies in surveillance of dysplasia in ulcerative colitis patients with high risk of colorectal cancer. Intest Res 2016;14:264-269.

2. Colman RJ, Rubin DT. Histological inflammation increases the risk of colorectal neoplasia in ulcerative colitis: a systematic review. Intest Res 2016;14:202-210.
3. Bryant RV, Burger DC, Delo J, et al. Beyond endoscopic mucosal healing in UC: histological remission better predicts corticosteroid use and hospitalisation over 6 years of follow-up. Gut 2016;65:408-414

4. Zenlea T, Yee EU, Rosenberg L, et al. Histology grade is independently associated with relapse risk in patients with ulcerative colitis in clinical remission: a prospective study. Am J Gastroenterol 2016;111:685-690.

5. Narang V, Kaur R, Garg B, et al. Association of endoscopic and histological remission with clinical course in patients of ulcerative colitis. Intest Res 2018;16:55-61.

6. Geboes K, Riddell R, Ost A, Jensfelt B, Persson T, Löfberg R. A reproducible grading scale for histological assessment of inflammation in ulcerative colitis. Gut 2000;47:404-409.

7. Mosli MH, Feagan BG, Zou G, et al. Development and validation of a histological index for UC. Gut 2017;66:50-58.

8. Mosli MH, Feagan BG, Sandborn WJ, et al. Histologic evaluation of ulcerative colitis: a systematic review of disease activity indices. Inflamm Bowel Dis 2014;20:564-575.

9. Marchal-Bressenot A, Salleron J, Boulagnon-Rombi C, et al. Development and validation of the Nancy histological index for UC. Gut 2017;66:43-49.

10. Christensen B, Hanauer SB, Erlich J, et al. Histologic normalization occurs in ulcerative colitis and is associated with improved clinical outcomes. Clin Gastroenterol Hepatol 2017;15:15571564.e1. 